\title{
A SINGLE-SIDEBAND OPTICAL BALANCED MIXED
}

\author{
Th. De GRAAUW, H. NIEUWENHUIJZEN, H. Van de STADT, R. GOEBEL * \\ The Astronomical Institute, Zonnenburg 2, Utrecht, The Netherlands
}

Received 5 March 1975

An optical single-sideband mixer, operating at $6328 \AA$ and utilizing two balanced heterodyne mixers, has been constructed. The sideband suppression ratio was measured to be typically about $25 \mathrm{~dB}$ and an optimal ratio of $54 \mathrm{~dB}$ was achieved.

Optical heterodyne detection can be used for high-resolution spectroscopy $[1,2]$. In this method, the radiation of the object under study is mixed with coherent laser local oscillator radiation in a square-law photodetector. The difference-frequency generated originates from two narrow spectral bands, equally spaced on both sides of the central laser frequency. In order to obtain unambiguous spectral information, for instance of an asymmetric line, one of these spectral bands has to be suppressed. In this paper we describe a so called single-sideband balanced mixer, operating at $6328 \AA$, based on a technique analogous to that which is frequently used in radiospectrum analysis [3] .

A description of the principle of an optical balanced mixer has been given by Ross [4]. Its practical advantages, elimination of laser amplitude noise and lossless combination of laser and signal beams, have been verified experimentally by Van de Stadt et al. [5] .

Fig. 1 shows the block diagrams of the experimental setup. It is essentially the heterodyne interferometer configuration as described by Van de Stadt [6], but with a slight modification. The signal and local oscillator beams are combined on four photodiodes with four half-reflecting, half-transmitting mirrors. For such lossless mirrors the reflection and transmission coefficients are $\mathrm{e}^{\mathrm{i} \pi / 4} / \sqrt{2}$ and $\mathrm{e}^{-\mathrm{i} \pi / 4} / \sqrt{2}$, respectively. The instantaneous power of the laser beam, $P_{0}$, and of the "right-hand side" and "left-hand side" signal beams $P_{\mathrm{r}}$ and $P_{\ell}$, respectively, at the input of the interferometer can be written as

$P_{0}=\frac{1}{2}\left[A_{0} \cos \left(\omega_{0} t+\varphi_{0}\right)\right]^{2}, \quad P_{\mathrm{r}}=\frac{1}{2}\left[A_{\mathrm{r}} \cos \left\{\left(\omega_{0}+a\right) t+\varphi_{\mathrm{r}}\right\}\right]^{2}, \quad P_{\ell}=\frac{1}{2}\left[A_{\ell} \cos \left\{\left(\omega_{0}-a\right) t+\varphi_{\ell}\right\}\right]^{2}$, where $\omega_{0}$ is the laser frequency, $a$ the difference frequency, $A_{0}, A_{\mathrm{r}}$ and $A_{\ell}$ the relevant amplitudes, and $\varphi_{0}, \varphi_{\mathrm{r}}$ and $\varphi_{Q}$ the corresponding phase angles. Having passed the beam splitters the radiation intensities received by the four photocells PC1, PC2, PC3 and PC4, can be written as

$P_{1}=\frac{1}{2}\left[A_{0} \cos \left(\omega_{0} t+\omega_{0}\right)+A_{\mathrm{T}} \cos \left\{\left(\omega_{0}+a\right) t+\varphi_{\mathrm{r}}-\frac{1}{2} \pi\right\}+A_{\ell} \cos \left\{\left(\omega_{0}-a\right) t+\varphi_{\ell}-\frac{1}{2} \pi\right\}\right]^{2}$,

$P_{2}=\frac{1}{2}\left[A_{0} \cos \left(\omega_{0} t+\varphi_{0}-\frac{1}{2} \pi\right)+A_{\mathrm{r}} \cos \left\{\left(\omega_{0}+a\right) t+\varphi_{\mathrm{r}}\right\}+A_{\ell} \cos \left\{\left(\omega_{0}-a\right) t+\varphi_{\ell}\right\}\right]^{2}$,

$P_{3}=\frac{1}{2}\left[A_{0} \cos \left(\omega_{0} t+\varphi_{0}+\frac{1}{2} \pi\right)+A_{\mathrm{r}} \cos \left\{\left(\omega_{0}+a\right) t+\varphi_{\mathrm{I}}+\frac{1}{2} \pi\right\}+A_{\ell} \cos \left\{\left(\omega_{0}-a\right) t+\varphi_{\ell}+\frac{1}{2} \pi\right\}\right]^{2}$,

$P_{4}=\frac{1}{2}\left[A_{0} \cos \left(\omega_{0} t+\varphi_{0}\right)+A_{\mathrm{r}} \cos \left\{\left(\omega_{0}+a\right) t+\varphi_{\mathrm{r}}+\pi\right\}+A_{\ell} \cos \left\{\left(\omega_{0}-a\right) t+\varphi_{\ell}+\pi\right\}\right]^{2}$.

\footnotetext{
* Now at Dudley Observatory, Albany, N.Y., USA.
} 


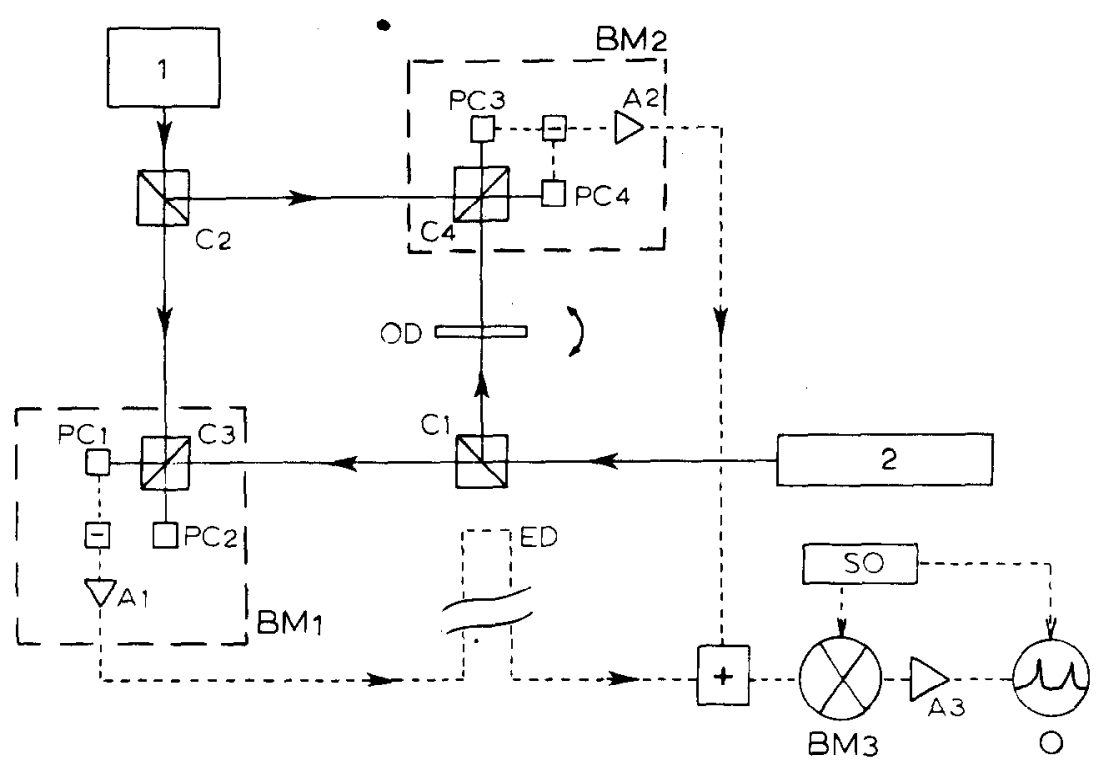

Fig. 1. Schematic diagram of the experimental setup of a single sideband optical balanced mixer. 1) Local oscillator laser; 2) signal laser; $\mathrm{C}_{1}, \mathrm{C}_{2}, \mathrm{C}_{3}, \mathrm{C}_{4}$ beam combiners; $\mathrm{PC}_{1}, \mathrm{PC}_{2}, \mathrm{PC}_{3}, \mathrm{PC}_{4}$, photocells; $\mathrm{BM}_{1}, \mathrm{BM}_{2}, \mathrm{BM}_{3}$ balanced mixers, OD optical delay, ED electronic delay; $A_{1}, A_{2}$ r.f. amplifiers, $A_{3}$ l.f. amplifier, SO signal sweep oscillator; $O$ oscilloscope.

Here we have taken into account the extra phaseshift of $\frac{1}{2} \pi$ in the signal beams for the two photocells $\mathrm{PC}_{3}$ and $\mathrm{PC}_{4}$. At the output of the balanced mixer BM1, constituted by the photocells PC1 and PC2, we shall obtain an r.f. current $i_{1}$ proportional to $P_{1}-P_{2}[4,5]$. The r.f. output current $i_{2}$ of the balanced mixer BM2 will be proportional to $\boldsymbol{P}_{\mathbf{3}}-\boldsymbol{P}_{\mathbf{4}}$. Neglecting terms with frequencies much larger than the r.f. bandpass frequency, we can write

$i_{1} \sim A_{0} A_{\mathrm{r}} \sin \left(a t+\varphi_{\mathrm{r}}-\varphi_{0}\right)-A_{0} A_{\ell} \sin \left(a t+\varphi_{0}-\varphi_{\ell}\right), \quad i_{2} \sim A_{0} A_{\mathrm{r}} \cos \left(a t+\varphi_{\mathrm{r}}-\varphi_{0}\right)+A_{0} A_{\ell} \cos \left(a t+\varphi_{0}-\varphi_{\ell}\right)$. After introducing a $\frac{1}{2} \pi$ phase delay in the signal coming from BMl, $i_{1}$ is changed into $i_{1}^{\prime}$

$i_{1}^{\prime} \sim A_{0} A_{\mathrm{r}} \cos \left(a t+\varphi_{\mathrm{r}}-\varphi_{0}\right)-A_{0} A_{\ell} \cos \left(a t+\varphi_{0}-\varphi_{\ell}\right)$.

Adding the currents $i_{1}^{\prime}$ and $i_{2}$ gives

$i_{1}^{\prime}+i_{2} \sim A_{0} A_{\mathrm{r}} \cos \left(a t+\varphi_{\mathrm{r}}-\varphi_{0}\right)$,

and their difference is

$i_{1}^{\prime}-i_{2} \sim A_{0} A_{Q} \cos \left(a t+\varphi_{0}-\varphi_{\ell}\right)$.

Phase shifting $i_{1}$ by $\frac{3}{2} \pi$ and adding it to $i_{2}$ or phase shifting $i_{2}$ by $\frac{1}{2} \pi$ and adding it to $i_{1}$ will also give expressions independent of $A_{\mathrm{r}}$. Hence we can determine $A_{\mathrm{r}}$ and $A_{\ell}$ separately.

In the experiment (see fig. 1) we have used a tunable single-mode He-Ne laser (Spectra Physics model 119) as a local oscillator. A second He-Ne laser (Spectra Physics model 122), operating in three axial modes also at $6328 \AA$, acted as a singal source [2]. The mode spacing for this laser is $385 \mathrm{MHz}$. The optical delay was introduced by a thin $(0.04 \mathrm{~mm})$ plane parallel glass plate [3] and could be adjusted by rotating this plate around a vertical axis. The electronic $\frac{1}{2} \pi$ phase delay was achieved by an additional coaxial cable introduced into one leg of the interferometer producing an electrical length difference of $\frac{1}{4} \lambda_{\text {r.f. }}$ where $\lambda_{\text {r.f. }}$ is the wavelength of the r.f. signal. The output signals $i_{1}^{i}$ and $i_{2}$ are combined in a threeport hybrid and led to an electronic balanced mixer, the local oscillator input of which is provided by a signal sweep oscillator operating between $0.1 \mathrm{MHz}$ and $500 \mathrm{MHz}$. The mixing signals were filtered $(1 \mathrm{kHz}-100 \mathrm{kHz})$, amplified and rectified. The resultant spectra were then displayed on an oscilloscope. 


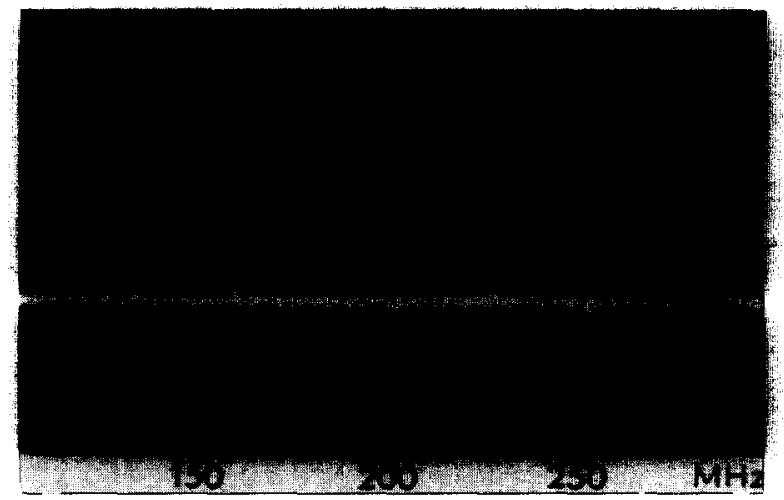

Fig. 2a. Oscilloscope display showing the left-hand sideband at $160 \mathrm{MHz}$ and the suppressed right thand sideband at $225 \mathrm{MHz}$. The signal at $130 \mathrm{MHz}$ is a result of the $385 \mathrm{MHz}$ beat signal of the multimode laser.

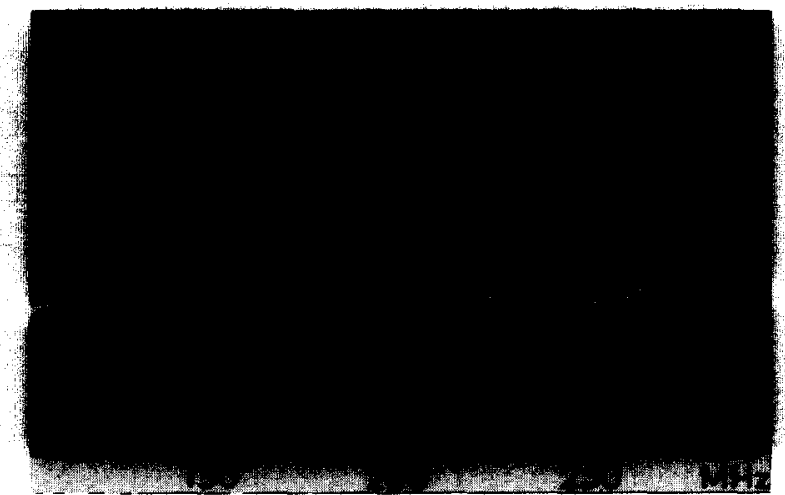

Fig. 2b. The suppressed sideband shows up again when one interferometer leg has been blocked.

The measurements were carried out in the following steps. First the L.O. laser was tuned between two modes of the signal laser. Next the electronic delay (the extra cable length) was inserted and the optical delay rotated until the amplitude of one of the sidebands was attenuated as far as possible and then the sideband suppression ratio was measured. Fig. 2a shows a result of this suppression. After inserting an optical stop into one leg of the interferometer the suppressed sideband showed up again as indicated in fig. $2 \mathrm{~b}$. Both sidebands are now weaker compared with the intensities in the initial situation because about one half of the radiation signal power is blocked. The experiment was repeated with different extra cable lengths, thus for different beat-frequencies. We have also checked the possibility of suppressing the other band by inserting delays in the appropriate r.f. lines as indicated above.

Temperature changes and acoustic noise caused much trouble in keeping the frequencies of the two lasers constant with relation to each other. They also prevented us to keep the optical delay constant over long periods of time. This problem will need much attention when building an equipment for routine measurements.

The sideband suppression ratio was measured to be typically about $25 \mathrm{~dB}$ and an optimal ratio of $54 \mathrm{~dB}$ was

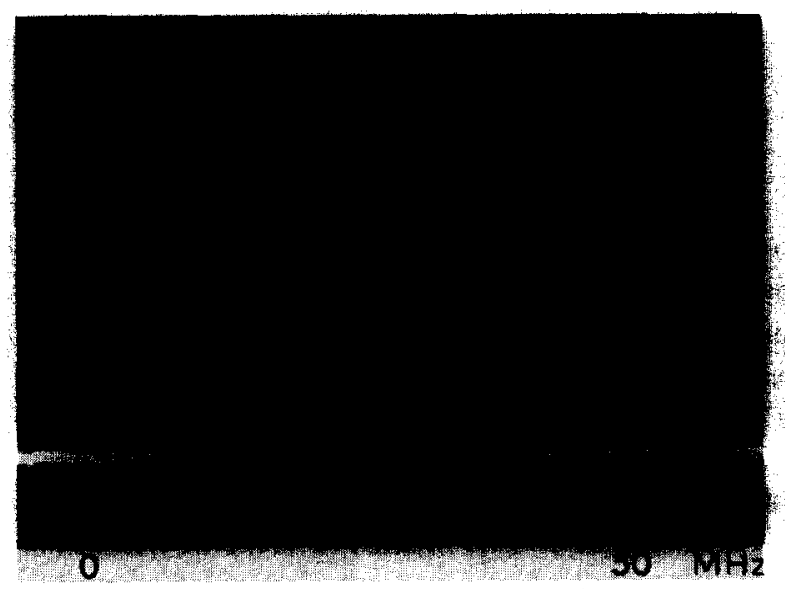

Fig. 3a. Sideband signal at $42 \mathrm{MHz}$.

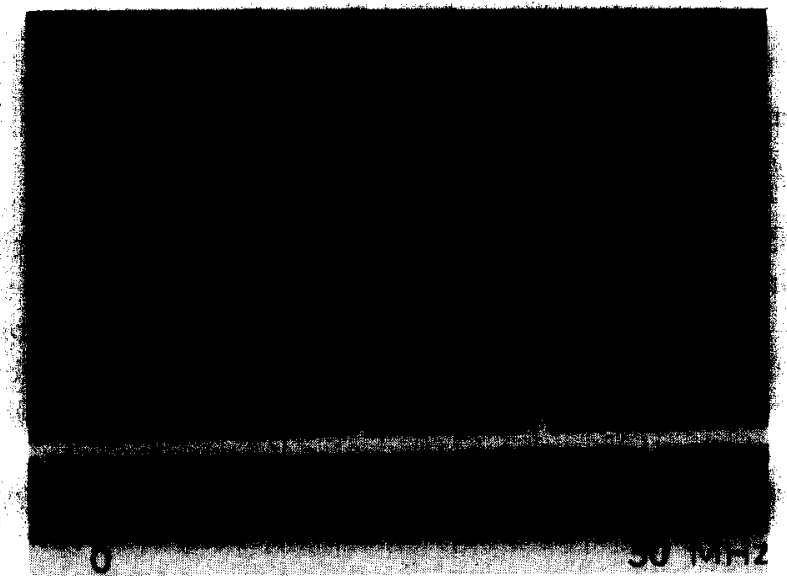

Fig. 3b. Result of the suppression of the $42 \mathrm{MHz}$ sideband. 
reached as shown in fig. $3 \mathrm{a}$ and $3 \mathrm{~b}$. This compares favourably with the results of Bakumenko and Antipenko [7] in an earlier experiment with single detectors.

Our experiments clearly demonstrate that suppression of one of the sidebands in an optical balanced mixer can be made very efficient and comparable to the results obtained by radio-astronomical techniques [8]. Sideband suppression over a large bandwidth $(10 \mathrm{MHz}-1000 \mathrm{MHz})$ requires a broad-band electronic delay. Since this problem has been resolved [9], heterodyne detection can indeed be used for high-resolution investigations of complex spectra.

We thank Professor H.G. van Bueren for stimulating this work. We acknowledge clarifying discussions with K. Wellington and J. Casse of the radio observatory at Dwingeloo, The Netherlands.

This work has been supported by the Netherlands Organization for the Advancement of Pure Research (Z.W.O.) and by the Netherlands Commission for Space Science and Geophysics (G.R.O.C.).

\section{References}

[1] D.W. Petersen, M.A. Johnson and A.L. Betz, Nature 250 (1974) 128.

[2] M. Mumma, T. Kostiuk, S. Cohen, D. Buhl and P.O. von Thuna, Proc. Rhenen Workshop on Coherent Detection in Astronomy (1974).

[3] D.E. Norgaard, Proc. I.R.E. 44 (1956) 1718.

[4] M. Ross, Laser Receivers (Wiley, 1965).

[5] H. Van de Stadt, Th. De Graauw, J.C. Shelton and Ch. Veth, Space Optics, Nat. Acad. Sc., Washington D.C. (1974) 443.

[6] H. Van de Stadt, Opt. Commun. 2 (1970) 153.

[7] V.M. Bakumenko and A.P. Antipenko, Radio Eng. and Electronic Phys. 17 (1972) 262 (Russian original 1971 ).

[8] A.E. Rogers, Proc. I.E.E.E. 39 (1970) 1617.

[9] K. Wellington, private communication. 